\title{
A Genetic Algorithm for Generating RF Circuit Models from Calibrated Broadband Measurements"
}

\author{
T. Mitch Wallis
}

\author{
Electromagnetics Division, National Institute of Standards and Technology, Boulder, Colorado, USA 80305
}

\begin{abstract}
A genetic algorithm has been developed to generate circuit models from calibrated broadband measurements of coaxial and on-wafer devices under test (DUTs). The algorithm randomly builds a population of circuit models from lumped as well as distributed elements and compares the simulated scattering parameters for those models with calibrated measurements. The genetic approach iteratively culls the circuit models that agree most closely with calibrated measurements. In order to "test-drive" this approach, the genetic algorithm was applied to calibrated measurements of several two-port $1.85 \mathrm{~mm}$ coaxial DUTs. The resulting circuit models are reasonable and their simulated response agrees closely with the measurements. Subsequently, the algorithm was applied to calibrated measurements of a two-port, on-wafer nanowire device. This algorithm automates exploration of potential circuit models for yet-to-be-modeled devices, including those that incorporate nanowires.
\end{abstract}

Index Terms - Calibration, Genetic algorithms, Microwave measurements, Nanowires, On-wafer devices.

\section{INTRODUCTION}

There is ongoing interest in the development of novel radio frequency $(\mathrm{RF})$ devices that incorporate emerging materials. Calibrated broadband measurements of such devices are often used to evaluate and optimize the devices. Additionally, such measurements may also reveal electromagnetic properties of the constituent materials themselves. This may be particularly important in a research and development environment in which there may be strong batch-to-batch variation in the constituent materials and in which material properties may be altered by fabrication processes. Moreover, RF measurements may reveal electromagnetic properties that are not accessible through other techniques, such as DC electrical or optical measurements. For example, the frequency-dependent, complex permittivity of ferroelectric, ferromagnetic, and multiferroic thin films has been extracted from broadband measurements of interdigitated capacitors and coplanar waveguides that incorporate those thin-film materials [1]. Several characteristic parameters of individual carbon nanotubes, such as the kinetic inductance and electrostatic contact capacitance, have been extracted from RF measurements [2]. Similarly, analysis of RF measurements of a nanowire-based, in-line RF transistor has been used to determine parasitic parameters of the device.

In general, the examples above rely on the development of RF circuit models of the devices under test (DUTs). In developing such models, one must consider the device design as well as the underlying materials physics that governs the device behavior. Ideally, the models will also be validated through analysis as well as circuit-based and finite-element simulations [4], [5]. However, even with rigorous validation, there is still considerable art to the development of the "best" circuit model. In some instances, it may be sufficient to simply require that the circuit model is consistent with measurements. If one wishes to extract circuit parameters that correspond to physical variables, such as the kinetic inductance of a carbon nanotube [6] or the contact impedance of a nanowire [7], it is necessary to incorporate such variables into the model. It is often desirable to keep a model simple in the sense that the model incorporates only those elements that are necessary and sufficient to capture the essential behavior of the DUT. Of course, an exhaustive search of all the possible models that might reproduce the measured device behavior is impractical. Here, we present a genetic algorithm that generates RF circuit models from calibrated measurements and automates the exploration of the circuit model parameter space. This algorithm is not intended to replace, but rather to complement the development and rigorous validation of circuit models through the application of researchers' experience and knowledge. Below, we describe the algorithm, test the algorithm's ability to reasonably model simple $1.85 \mathrm{~mm}$ coaxial DUTs, and apply the algorithm to a two-port, onwafer, GaN nanowire device.

\section{THE GENETIC ALGORITHM}

Genetic algorithms are a biologically inspired, iterative approach to complicated optimization problems [8]. Genetic algorithms have been widely used to design and model RF circuits [9], [10]. Two-port error boxes have been determined from a broadband measurement of a thru by use of a genetic algorithm [11]. Models of nonlinear devices have been generated from large-signal measurements by use of neural 
networks, which are an alternative biologically inspired approach [12]. Genetic algorithms are well suited for developing circuit models from calibrated RF measurements as the problem is unlikely to have a unique global solution, i.e. there are a multitude of different models that may be consistent with the calibrated, measured data.

A genetic algorithm to generate simple RF circuit models from calibrated broadband measurements of coaxial and onwafer DUTs has been developed and implemented through custom software written in $\mathrm{C}++$. A key choice that must be made at the outset is the determination of how the circuit parameters will be represented. Here, each circuit model consisted of six cascaded elements. The identity of the elements, as well as the parameter values, were encoded in strings of eight-bit numbers. A multi-step process simulated the scattering parameters of each circuit model: (1) an ABCD matrix was generated for each of the six elements (2) the six ABCD matrices were cascaded (3) the resulting ABCD matrix was transformed to a scattering parameter representation. The circuits generated by the algorithm are particularly sensitive to which circuit elements are available as building blocks for the models. Our ultimate goal here is to model nanowire-based, two-port devices. Thus, based on circuit models of such devices in the literature [2]-[5], we included lumped elements such as series resistors and shunt capacitors, distributed transmission line elements, as well as a thru circuit element. The choice of the circuit representation implies a number of assumptions that must be met by the modeled DUT, including that the device be linear and passive.

Initially, the algorithm builds a population of circuit models by randomly selecting the circuit elements and parameter values for each model. In the experiments described below, the population size was chose to be sixteen models. The scattering parameters that correspond to the circuit are then simulated and compared to the scattering parameters obtained from calibrated measurements. The genetic approach culls the best circuit models from the population by selecting those models that minimize the following cost function:

$$
c=\sum_{i, j} \sum_{N} k_{i j}\left(\left|\Delta_{r}\left(f_{N}\right)\right|+\left|\Delta_{i}\left(f_{N}\right)\right|\right),
$$

where

$$
\Delta_{r}\left(f_{N}\right)=\operatorname{real}\left(S_{i j}^{\text {meas }}\left(f_{N}\right)\right)-\operatorname{real}\left(S_{i j}^{\text {sim }}\left(f_{N}\right)\right)
$$

and

$$
\Delta_{i}\left(f_{N}\right)=\operatorname{imag}\left(S_{i j}^{\text {meas }}\left(f_{N}\right)\right)-\operatorname{imag}\left(S_{i j}^{\operatorname{sim}}\left(f_{N}\right)\right) .
$$

In (1), (2), and (3), $i$ and $j$ are the scattering parameter indices ( $i=1,2 ; j=1,2)$, the set of $f_{N}$ are the frequencies at which the measurements were taken, $\operatorname{real}\left(S_{i j}^{s i m}\right)$ and $\operatorname{imag}\left(S_{i j}^{s i m}\right)$ are the real and complex parts of the simulated scattering parameters, and $\operatorname{real}\left(S_{i j}^{\text {meas }}\right)$ and $\operatorname{imag}\left(S_{i j}^{\text {meas }}\right)$ are the real and complex parts of the calibrated measured scattering parameters. In the discussion below, the weighting coefficients $k_{i j}$ were all kept equal to one, expect where indicated otherwise. The algorithm generates new circuit models by "mating" and "mutating" the circuit models with the lowest cost $c$ in order to produce a new generation of models. By repeating this process for many cycles, the algorithm explores the circuit model parameter space and over time generates a population of circuit models that are in good agreement with the calibrated measurements. The algorithm terminates when the average cost $c$ of the population of circuit models is equal to or less than a target cost set by the user.
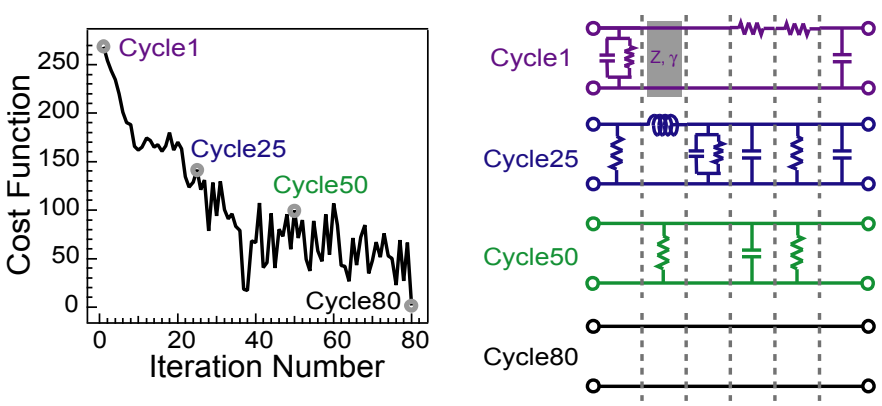

Fig. 1. (Left Panel) Cost history of the population of circuit models for the $1.85 \mathrm{~mm}$ coaxial thru. (Right Panel) Circuit models with the lowest cost at selected iteration cycles throughout the process.

\section{MODELING OF 1.85 MILLIMETER COAXIAL DEVICES}

Before applying the algorithm to nanowire-based devices, we tested the algorithm on several $1.85 \mathrm{~mm}$ coaxial devices for which relatively simple, physically reasonable circuit models have already been established. The objective is twofold: (1) to confirm that the algorithm generates reasonable circuit models for the coaxial devices, within the constraints of the available circuit elements and (2) to explore the efficiency and potential limitations of the approach. The measurements were made with a commercial vector network analyzer (VNA). The standards used to calibrate the VNA include a set of $1.85 \mathrm{~mm}$ air lines, a thru, a pair of flat shorts, and a pair of matched loads. The calibration analysis was carried out with NIST's MultiCal software. Three DUT configurations were considered: a thru, a matched load on each port, and an offset short on each port. The loads and offset shorts were taken from a commercial calibration kit.

The average cost of the thru circuit model population as a function of iterative cycle is shown in Fig. 1, along with schematic representations of the best circuit model in the population at various iterative cycles in the process. Note that in Fig. 1, the circuit models are not in their simplest form; resistors in series have not been combined into a single resistor, for example. The circuit from the original cycle (Cycle 1) is a random collection of six lumped and distributed circuit elements. As the cycles, continue, there are an increasing number of thru elements in the circuit, until the 
entire circuit consists of six thru elements in the final iteration (Cycle 80). A comparison between the simulated scattering parameters of the circuits in Fig. 1 and the calibrated measurements is shown in Fig. 2. Note that while the overall trend is an improvement in the agreement between the simulated and measured data, the average cost may occasionally increase from one given cycle to the next.

Four different circuit models for the pair of loads were obtained. Typically, these models were obtained within about one thousand iterations. On a desktop PC running a commercial operating system, one thousand iterations require a few minutes of computation. One of the obtained models is shown in Fig. 3 while the simulated scattering parameters for four different models are shown in Fig. 4, along with the calibrated measurements. Each of the four models agrees reasonably well with the measurements, though none of the models fully capture the oscillatory behavior in the real and imaginary parts of $S_{I I}$. Clearly, the algorithm will find different solutions on separate runs, rather than a single global solution every time. Thus, repeated runs may enable the user to compare and contrast different circuit model options that are consistent with the measured data. Examination of the circuit diagram for the pair of loads in Fig. 3 also reveals several notable features. First, there is a $\sim 50 \bullet$ shunt across each port. Within the constraints of the circuit elements available in this algorithm, this is a physically reasonable

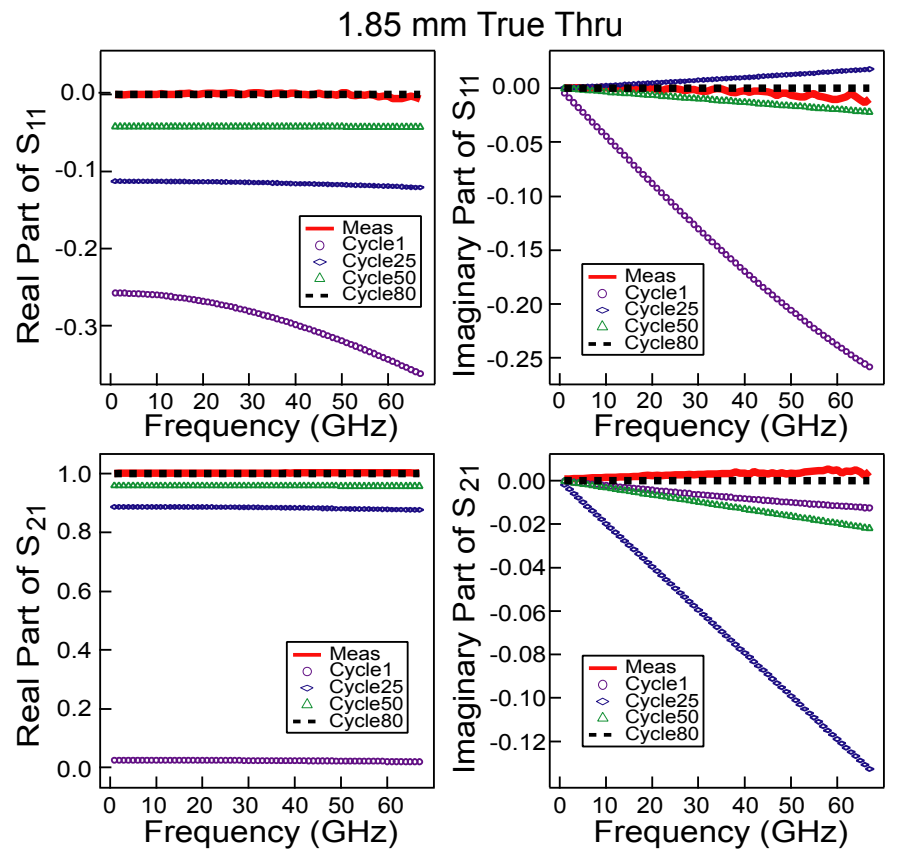

Fig. 2. Simulated scattering parameters of the circuit models of the $1.85 \mathrm{~mm}$ thru shown in Fig. 1 compared to calibrated measurements. $S_{22}$ and $S_{12}$ (neither shown) are comparable to $S_{11}$ and $S_{21}$, respectively. The uncertainty in the calibrated measurements of the scattering parameters is estimated to be $+/-0.005$. representation of a matched load, though the actual internal implementation is likely to be different than a 50 - resistor. Note that a small $(0.13 \mathrm{fF})$ series capacitor effectively separates the two ports. Finally, there is a $0.67 \mathrm{fF}$ shunt capacitor across port two, which is somewhat difficult to interpret. This sort of extra feature occasionally appears in the circuits found by this algorithm, but the parameter values are usually in such a range that their influence on the overall performance of the circuit is minimal. Further analysis is required to determine if such an element is the result of imperfect DUTs, user error in the calibrated measurement process, or a "vestigial" feature that may be simply ignored.

A schematic of a circuit model obtained for the pair of offset shorts is also shown in Fig. 3. The simulated scattering parameters for that model are shown in Fig. 5, along with the calibrated measurements. The simulated scattering parameters are in good agreement with the measurements. Note that the circuit model includes transmission line segments on either port, while small shunt inductors on either port effectively simulate the short circuit. As with the load a very small series capacitor effectively separates the two ports. Within the constraints of the available circuit elements, this is a physically reasonable representation of offset shorts, though once again there is a "vestigial" element: a relatively small (6.45 fF) shunt capacitance across port one.

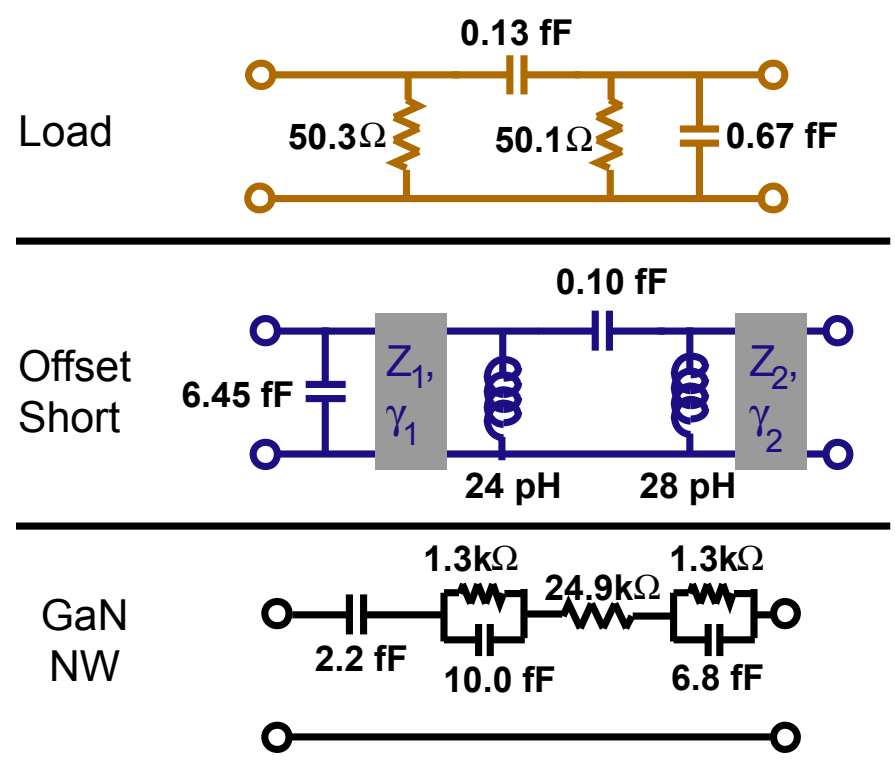

Fig. 3. Circuit models obtained for a pair of loads, a pair of offset shorts, and a GaN NW device. Note that the circuits have been simplified from their six-element representations, where appropriate.

\section{MODELING OF A GAN NANOWIRE DEVICE}

Having gained some confidence in the ability of the algorithm to generate reasonable circuit models, we applied the algorithm to a two-port $\mathrm{GaN}$ nanowire (NW) device that consists of a $4 \mu \mathrm{m}$-long GaN NW integrated into a coplanar 


\section{$1.85 \mathrm{~mm}$ Loads}
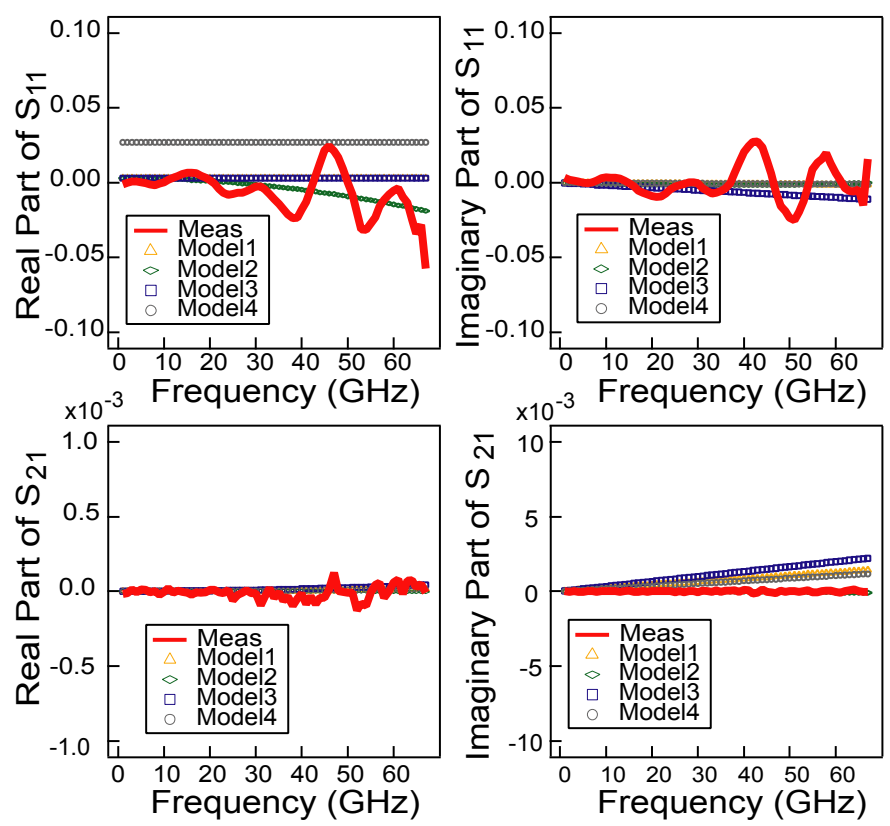

Fig. 4. Simulated scattering parameters of the circuit models of $1.85 \mathrm{~mm}$ loads compared to calibrated measurements. $\mathrm{S}_{22}$ and $\mathrm{S}_{12}$ (neither shown) are comparable to $S_{11}$ and $S_{21}$, respectively.

\section{$1.85 \mathrm{~mm}$ Offset Shorts}
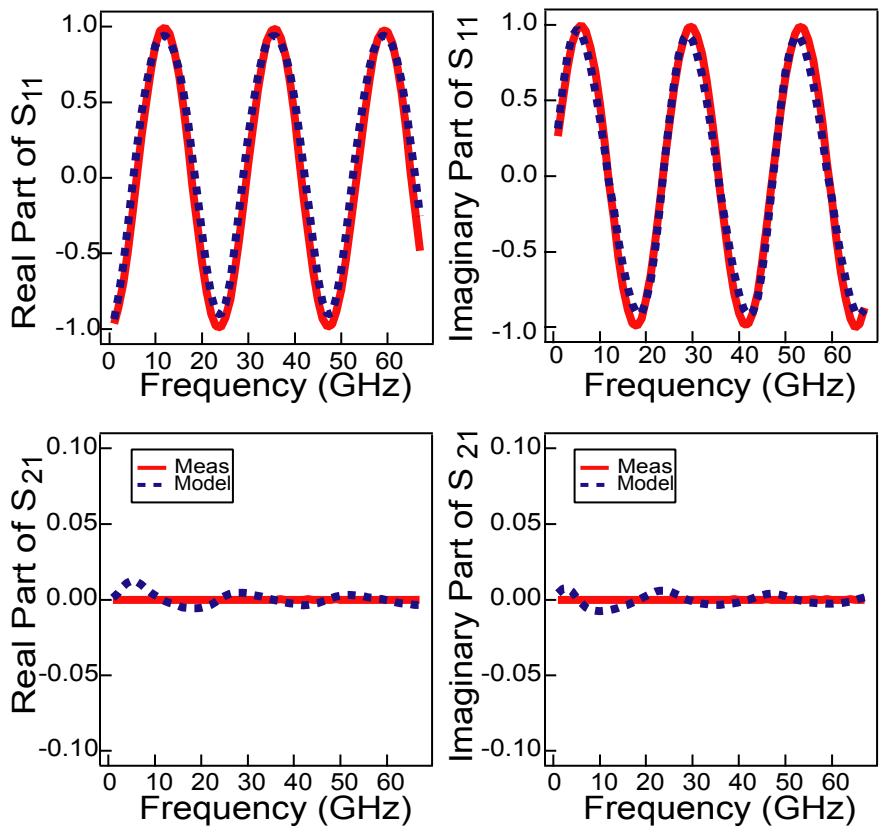

Fig. 5. Simulated scattering parameters of the circuit models of $1.85 \mathrm{~mm}$ offset shorts compared to calibrated measurements. $\mathrm{S}_{22}$ and $\mathrm{S}_{12}$ (neither shown) are comparable to $\mathrm{S}_{11}$ and $\mathrm{S}_{21}$, respectively. waveguide host structure. The fabrication and calibrated measurement process of the two-port GaN NW device has been described in detail elsewhere [5], [13]. A schematic of a circuit model obtained for the GaN NW Device is shown in Fig. 3 while the simulated scattering parameters for that model are shown in Fig. 6, along with the calibrated measurements. It was empirically found that the cost function could be best minimized if the weighting coefficients were set to $k_{12}=k_{21}=2$ and $k_{11}=k_{22}=1$. Because of this choice in weighting coefficients however, some agreement with the reflection terms, particularly the complex part of $S_{11}$ and $S_{22}$, has been sacrificed. Several elements of the circuit easily lend themselves to physical interpretation. The central $24.9 \mathrm{k} \cdot$ resistor may be interpreted as the intrinsic resistance of the NW. The combinations of $1.3 \mathrm{k} \cdot$ resistances in parallel with capacitances may be interpreted as the contacts to the NW. In previous work [13], we have assumed that the contact impedance was purely resistive, but the model developed here suggests that there may be a non-negligible electrostatic capacitance associated with the contacts. Finally, there is a series capacitor that may be interpreted in multiple ways. Note that this capacitor may have been introduced by the algorithm to generally suppress transmission through this device, which is clearly poorly matched to the test equipment. Alternatively, this capacitor may indicate that one of the contacts is not ohmic. Though the device was annealed in order to produce ohmic contacts, this suggests that there might still be a small Schottky capacitance associated with the contacts. Further measurements with varying DC bias on the device would be required to confirm this hypothesis.

\section{CONCLUSION}

The genetic algorithm is capable of generating a population of circuit models from calibrated measurements of yet-to-bemodeled devices. Study of such a population of alternative models may complement the development and validation of models through more traditional approaches. On the whole, the circuit models that were developed for the $1.85 \mathrm{~mm}$ coaxial DUTs and the two-port GaN NW device were in good agreement with calibrated measurements and were physically reasonable representations of the various DUTs within the constraints of the number and types of circuit elements that were available. However, there is still room for improvement. For example, improved mating and mutation strategies may allow the algorithm to explore the circuit model parameter space more effectively. Additions to or refinements of the circuit elements that are available within the circuit representation may also lead to closer agreement with measurements. Porting the process to a more powerful computing system may also allow for a more efficient and lengthier search for circuit models that are in goo agreement with measured data (At present, the algorithm was run on a desktop PC that is several years old, running a commercial 
desktop operating system). Also, while this work is focused on the generation of a complete circuit model through the genetic approach, the approach may also be applied to unknown subsections of otherwise known devices, e.g. an established model of a NW may be held fixed while models of the contacts to the NW are explored via the genetic algorithm. Finally, it remains to be seen if the genetic algorithm may be successfully applied to un-calibrated measurements in order to obtain models of the error boxes as well as the DUTs.

\section{Two-Port GaN NW Device}
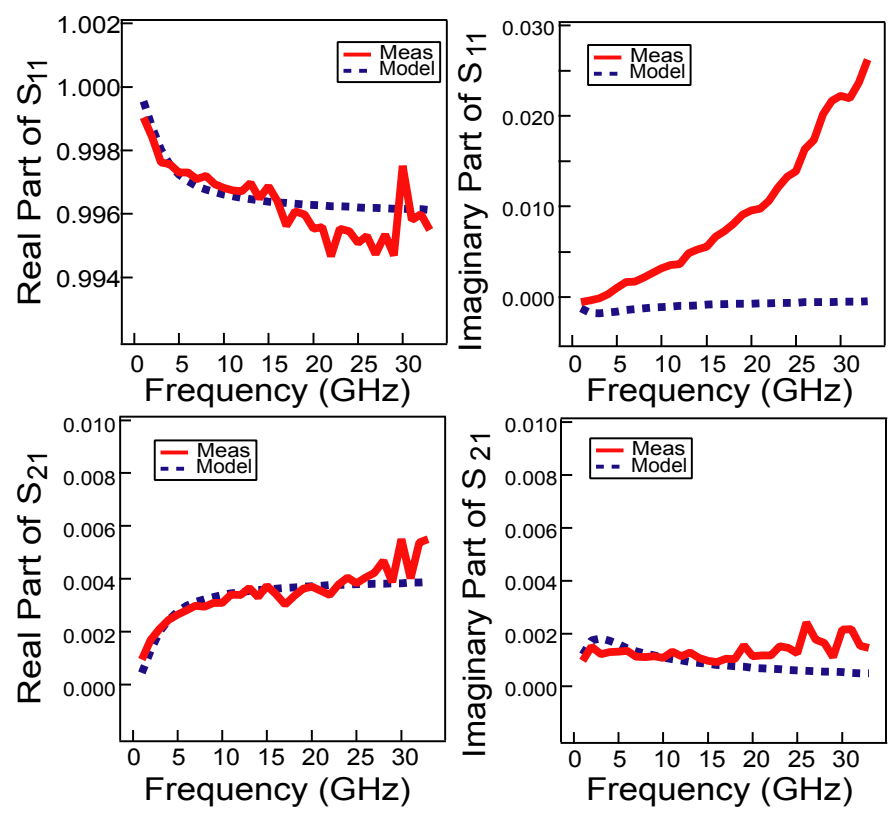

Fig. 6. Simulated scattering parameters of the circuit models of a two-port GaN NW device compared to calibrated measurements. $S_{22}$ and $S_{12}$ (neither shown) are comparable to $S_{11}$ and $S_{21}$, respectively.

\section{REFERENCES}

[1] N. Orloff, J. Mateu, M. Murakami, I. Takeuchi, and J. C. Booth, "Broadband characterization of multilayer dielectric thin-films," 2007 IEEE MTT-S International Microwave Symposium Digest (MTT), pp. 1177-1180, 2007.
[2] J. J. Plombon, K. P. O’Brien, F. Gstrein, V. M. Dubin, and Y. Jiao, "High-frequency electrical properties of individual and bundled carbon nanotubes," Appl. Phys. Lett., vol. 90, art. no. 063106, 2007.

[3] D. Gu, T. M. Wallis, P. Blanchard, S.-H. Lim, A. Imtiaz, K. A. Bertness, N. A. Sanford, and P. Kabos, "Deembedding parasitic elements of $\mathrm{GaN}$ nanowire metal semiconductor field effect transistors by use of microwave measurements," Appl. Phys. Lett., vol. 98, art. no. 223109, 2011.

[4] K. Kim, T. M. Wallis, P. Rice, C.-J. Chiang, A. Imtiaz, P. Kabos, and D. Filipovic, "Modeling and metrology of metallic nanowires with application to microwave interconnects," 2010 IEEE MTT-S International Microwave Symposium Digest (MTT), pp. 1292-1295, 2010.

[5] K. Kim, T. M. Wallis, P. Rice, C.-J. Chiang, A. Imtiaz, P. Kabos, and D. Filipovic, "A framework for broadband characterization of individual nanowires," IEEE Microwave and Wireless Component Lett, vol. 20pp. 178-180, 2010.

[6] C. Rutherglan and P. J. Burke, "Nanoelectromagnetics: circuit and electromagnetic properties of carbon nanotubes," Small, vol. 5, pp. 884-906, 2009.

[7] C.-J. Chiang, T. M. Wallis, D. Gu, A. Imtiaz, P. Kabos, P. T. Blanchard, K. A. Bertness, N. A. Sanford, K. Kim, and D. Filipovic, "High frequency characterization of a Schottky contact to a GaN nanowire bundle," Journal of Applied Physics, vol. 107, art. no. 124301, 2010.

[8] R. L. Haupt and S. E. Haupt, Practical Genetic Algorithms, New York: J. Wiley \& Sons, 1998.

[9] J. G. Pascual, P. F. Quesada, D. C. Rebenaque, J. L. G. Tornero, and A. A. Melcon, "A multilayered shielded microwave circuit design method based on genetic algorithms and neural networks," 2006 IEEE MTT-S International Microwave Symposium Digest (MTT), pp. 1427-1430, 2006.

[10] S. Iezekiel, "Application of evolutionary computation techniques to nonlinear microwave circuit analysis," $8^{\text {th }}$ IEEE International Symposium on High Performance Electron Devices for Microwave and Optoelectronic Applications, pp. 230-235, 2000.

[11] A. S. Adalev, N. V. Korovkin, M. Hayakawa, and J. B. Nitsch, "Deembedding and unterminating microwave fixtures with the genetic algorithm," IEEE Transactions on Microwave Theory and Techniques, vol. 54, pp. 3131-3140, 2006.

[12] J. Jargon, K. C. Gupta, D. Schreurs, K. Remley, D. DeGroot, “A method of developing frequency-domain models for nonlinear circuits based on large-signal measurements," $58^{\text {th }}$ ARFTG Conference Digest, pp. 35-48, 2001

[13] T. M. Wallis, D. Gu, A. Imtiaz, C. S. Smith, C.-J. Chiang, P. Kabos, P. T. Blanchard, N. A. Sanford, and K. A. Bertness "Electrical characterization of photoconductive GaN nanowire devices from $50 \mathrm{MHz}$, to $33 \mathrm{GHz}$," IEEE Transactions on Nanotechnology, vol. 10, pp. 832-838, 2011. 